\title{
Spin-polarization parameters and cross sections for electron scattering from zinc and lead atoms
}

\author{
Pradeep Kumar, Arvind Kumar Jain, and A. N. Tripathi \\ Department of Physics, University of Roorkee, Roorkee-247667 India \\ Sultana N. Nahar \\ Department of Astronomy, Ohio State University, Columbus, Ohio 43210
}

(Received 10 June 1993)

\begin{abstract}
A relativistic approach is employed to compute the differential and integrated cross sections, spinpolarization $P$, and the spin-polarization parameters $T$ and $U$ for the scattering of electrons from zinc and lead atoms in the energy range $2.0-200 \mathrm{eV}$. The projectile-target interaction is represented both by real and complex optical potentials in the solution of the Dirac equation for scattered electrons. We compare our results for spin-polarization $\boldsymbol{P}$ with recent experiment and available theoretical calculations.
\end{abstract}

PACS number(s): $34.80 . \mathrm{Bm}, 34.80 . \mathrm{Nz}$

\section{INTRODUCTION}

It is well known that for the elastic scattering of slow unpolarized electrons from heavier atoms, spin-orbit effects can produce detectable changes in the spin polarization of the scattered electrons. This process is often referred to as Mott scattering. Recent progress both in measurement techniques and in the availability of efficient sources of polarized electrons has led to considerable interest in such experiments. It is now possible to perform experiments in which a complete set of spinpolarization parameters can be measured. Such measurements thus provide direct information about spin-orbit $(l \cdot s)$ interaction, a weak interaction which is generally masked by the much stronger Coulomb interaction. The measurements of all the polarization parameters in electron-heavy-atom collision from low to intermediate impact energies have been reported by a number of groups. Measurements up to the year 1990 have been reviewed by Kessler [1]; older work can be traced through the review article of Hanne [2]. Mott [3] was the first to investigate the spin polarization of electrons scattered by a central potential using a relativistic treatment based on Dirac theory. Recent laboratory measurements have led to further studies of the spin-dependent electronscattering processes. Among the many early theoretical calculations, the work of Walker [4]; Sin Fai Lam [5]; Awe et al. [6]; Fritsche, Noffke, and Gollisch [7]; and Kemper, Rosicky, and Feder [8] are worth mentioning. Recently, McEachran and Stauffer [9], Hasenburg et al. [10], Nahar and Wadehra [11], and Nahar [12] calculated a complete set of polarization parameters for a number of heavier atoms using a relativistic approximation. In addition, Haberland and Fritsche [13], Bartschat [14], and Bartschat, Goerss, and Nordbeck [15] have also reported calculations for spin-dependent scattering processes using more sophisticated approaches such as the generalized Kohn-Sham (GKS) method and a static exchange $R$ matrix theory, respectively.

In addition to the pure spin-orbit interaction effect, po- larization of the scattered electron can also occur due to the interplay between the spin-orbit splitting of atomic fine-structure states, referred to as fine-structure effects. In elastic scattering from a closed-shell configuration, only Mott scattering need be considered, but for openshell systems, the fine-structure effect also becomes important. Recently, Bartsch et al. [16] have examined in detail the role of different spin-dependent interactions.

McEachran and Stauffer [9] and Nahar and Wadhera [11] both solved the relativistic form of the Schrödinger equation. In the former case, the static and relativistic potentials for atoms were obtained from relativistic Hartree-Fock wave functions; the polarization potentials from a nonrelativistic polarized orbital method and exchange were included exactly through the large component of the scattered wave function, while in the latter case the projectile-target interaction $V(r)$ is represented by both a real and a complex model potential. The real part of the interaction accounts only for the pure elastic scattering and is used to calculate the elastic-scattering cross sections and spin-polarization parameters. Further, these scattering parameters, along with total-scattering cross sections can also be obtained using the complex model potential. In this case, a model absorption potential $\boldsymbol{V}_{\mathrm{abs}}(\boldsymbol{r})$ is taken as the imaginary part of a total interaction that includes both elastic- and inelasticscattering processes.

Following Nahar and Wadehra [11], we have calculated spin-polarization parameters, differential cross sections (DCS), and integrated elastic, momentum-transfer, and total cross sections for the scattering of electrons from zinc and lead atoms using both real and complex potentials within the framework of the relativistic Dirac equation. Recently, Bartsch et al. [16] have reported experimental measurements on asymmetry functions for the elastic scattering of polarized electrons from zinc, cadmium, and indium atoms at low energy. In Sec. II we review briefly the theoretical methodology and the details of our approach. In Sec. III we present our results and compare them with existing experimental and available theoretical calculations. 


\section{THEORY}

\section{A. Theoretical methodology}

The Dirac equation for a projectile of rest mass $m_{0}$ traveling in a central field $V(r)$ at a velocity $v$ is given by

$$
\left[c \alpha \cdot \mathbf{p}+\beta m_{0} c^{2}+V(r)\right] \psi=E \psi,
$$

where $E=m_{0} \gamma c^{2}=E_{i}+m_{0} c^{2}$ is the total energy, $\gamma=\left(1-v^{2} / c^{2}\right)^{-1 / 2}$, and $E_{i}$ is the kinetic energy of the incident particle. $\alpha$ and $\beta$ are the usual $4 \times 4$ Dirac matrices. The spinor $\psi$ has the four components and $\psi=\left(\psi_{1}, \psi_{2}, \psi_{3}, \psi_{4}\right)$, where $\left(\psi_{1}, \psi_{2}\right)$ are "large" components and $\left(\psi_{3}, \psi_{4}\right)$ are "small" components of $\psi$. For a central potential, the Dirac equation can be reduced to a set of two equations [11],

$$
g_{l}^{ \pm \prime \prime}+\left[K^{2}-l(l+1) / r^{2}-U_{l}^{ \pm}(r)\right] g_{l}^{ \pm}(r)=0,
$$

where $g_{l}^{ \pm}$is related to the radial part $G_{l}^{ \pm}$of the large component of $\psi$ as

$$
\begin{aligned}
G_{l} & =\sqrt{\eta} \frac{g_{l}}{r}, \quad \eta=\frac{\left[E-V(r)+m_{0} c^{2}\right]}{c \hbar}, \\
K^{2} & =\frac{\left(E^{2}-m_{0}^{2} c^{4}\right)}{\hbar^{2} c^{2}} .
\end{aligned}
$$

The $U_{l}^{ \pm}$are the effective Dirac potentials and are given in atomic units $\left(m_{0}=e=\hbar=1,1 / c=\alpha\right.$, where $\alpha$ is the finestructure constant) as

$$
-U_{l}^{+}(r)=-2 \gamma V+\alpha^{2} V^{2}-\frac{3}{4} \frac{\left(\eta^{\prime}\right)^{2}}{\eta^{2}}+\frac{1}{2} \frac{\eta^{\prime \prime}}{\eta}+\frac{(l+1)}{r} \frac{\eta^{\prime}}{\eta}
$$

and

$$
-U_{I}^{-}(r)=-2 \gamma V+\alpha^{2} V^{2}-\frac{3}{4} \frac{\left(\eta^{\prime}\right)^{2}}{\eta^{2}}+\frac{1}{2} \frac{\eta^{\prime \prime}}{\eta}-\frac{l}{r} \frac{\eta^{\prime}}{\eta} .
$$

Here, single and double primes denote the first and second derivatives with respect to $r$, respectively. It should be noted that the last term of $U_{I}^{ \pm}$in Eqs. (3) and (4) corresponds to the two eigenvalues of the well-known spin-orbit interaction, one due to spin up and other due to spin down:

$$
\frac{1}{4 m_{0}^{2} c^{2}} \frac{1 d V(r)}{r d r} \sigma \cdot \mathrm{L} \text {. }
$$

In the nonrelativistic treatment of the Schrödinger equation, the above term is treated as a small perturbative term along with projectile-target interaction. Here, $\sigma$ is related to spin $S$ as $\sigma=2 S$ and the value of $(\sigma \cdot L)$ equals $l$ for $j=l+\frac{1}{2}$ and $-(l+1)$ for $j=l-\frac{1}{2}$. The proper solution of Eq. (2) behaves asymptotically as

$$
g_{l}^{ \pm}(K, r)=K r\left[j_{l}(K r)-\tan \left(\delta_{l}^{ \pm}\right) \eta_{l}(K r)\right], \quad r \rightarrow \infty
$$

where $j_{l}$ and $\eta_{1}$ are the spherical Bessel functions of the first and second kind, respectively. The phase shifts $\delta_{l}^{ \pm}$ can be obtained from the values of the radial wave function $g_{l}^{ \pm}$at the two adjacent points $r$ and $r+h(h \ll r)$ at very large $r$ as

$\tan \delta_{l}^{ \pm} \simeq-\frac{(r+h) g_{l}^{ \pm}(r) j_{l}[K(r+h)]-r g_{l}^{ \pm}(r+h) j_{l}(K r)}{r g_{l}^{ \pm}(r+h) \eta_{l}(K r)-(r+h) g_{l}^{ \pm}(r) \eta_{l}[K(r+h)]}$

In the present calculation, the wave functions $g_{l}^{ \pm}$are obtained by numerical integration of Eq. (2) using Numerov's method. In the present work, a large number of phase shifts are evaluated. For example, the typical values of $l$ corresponding to the impact energies 2.0 and $200 \mathrm{eV}$ are 15 and 50, respectively.

\section{B. Cross section and spin-polarization parameters}

The generalized scattering amplitude for the collision process is given as [17]

$$
A=f(K, \vartheta)+g(K, \vartheta) \sigma \cdot \hat{\mathbf{n}},
$$

where $f(K, \vartheta)$ and $g(K, \vartheta)$ are direct and spin-flip scattering amplitudes and are given as

$$
\begin{aligned}
f(K, \vartheta)=\frac{1}{2 i K} \sum_{l=0}^{\infty}[ & {[l+1)\left(e^{2 i \delta_{l}^{+}}-1\right) } \\
& \left.+l\left(e^{2 i \delta_{l}^{-}}-1\right)\right] p_{l} \cos (\vartheta)
\end{aligned}
$$

and

$$
g(K, \vartheta)=\frac{1}{2 K} \sum_{l=0}^{\infty}\left(e^{2 i \delta_{l}^{+}}-e^{2 i \delta_{l}^{-}}\right) p_{l}^{1} \cos (\vartheta)
$$

and $\hat{\mathbf{n}}$ is a unit vector perpendicular to the scattering plane. $p_{l}$ and $p_{l}{ }_{1}^{1}$ are regular and associated Legendre polynomials, respectively. The elastic differential cross section for the scattering of the unpolarized incident beam is given by

$$
d \sigma / d \Omega=|f|^{2}+|g|^{2},
$$

and the polarization produced in the unpolarized incident beam due to the scattering is given by

$$
P(\vartheta)=\frac{\left(f g^{*}+g f^{*}\right) \hat{\mathbf{n}}}{|f|^{2}+|g|^{2}}=P(\vartheta) \hat{\mathbf{n}}
$$

where $P(\vartheta)$ is the Sherman function, and two other spinpolarization parameters $T$ and $U$,

$$
\begin{aligned}
& T(\vartheta)=\frac{|f|^{2}-|g|^{2}}{|f|^{2}+|g|^{2}}, \\
& U(\vartheta)=i \frac{f g *-g f^{*}}{|f|^{2}+|g|^{2}} .
\end{aligned}
$$

In the case of elastic scattering, the spin asymmetry function is identical to the Sherman function $P(\vartheta)$.

\section{The interaction potential}

The total interaction between the projectile and the target atom is represented by a model potential. At the 
impact energies, above the first ionization threshold, this potential is complex and is written as

$$
V(r)=V_{R}(r)+i V_{\mathrm{abs}}(r),
$$

where $V_{R}(r)$ is the real part of the total interaction potential $V(r)$, is represented by three local real terms, namely, the static (due to direct Coulomb interaction of all the charged particle involved), exchange (arising due to the Pauli exclusion principle), the polarization (taking into account the distortion of the target charge cloud in the presence of an incoming electron field). $V_{\text {abs }}(r)$ is the absorption potential. Its inclusion parametrizes inelastic processes such as excitation, ionization, recombination, etc. The static potential of the target atom is obtained by averaging over the motion of the target electrons and is given as

$$
\begin{aligned}
V_{s}(r)= & \frac{Z e e_{p}}{r} \\
& -e e_{p} \sum_{n} \sum_{l} \sum_{m} N_{n l m} \int\left|\phi_{n l m}\left(\mathbf{r}^{\prime}\right)\right|^{2} \frac{1}{\left|\mathrm{r}-\mathrm{r}^{\prime}\right|} d \mathbf{r}^{\prime},
\end{aligned}
$$

where $Z$ is the nuclear charge of the target atom. $e_{p}$ is the projectile charge and $N_{n l m}$ is the occupancy number of the orbit $(n, l, m)$. The radial part $\varphi(r)$ of the spatial orbital

$$
\varphi_{n l m}(r)=\varphi_{n l}(r) y_{l m}(\hat{\mathbf{r}})
$$

is expanded in terms of Slater-type orbitals given in the tables of Clementi and Roetti [18] and McLean and McLean [19]. $V_{p}(r)$ in Eq. (17) is the parameter-free polarization potential and is taken from the paper of O'Connell and Lane [20]. It has two components, the short-range $\left[V_{S R}(r)\right]$ part and the long-range $\left[V_{L R}(r)\right]$ part and is given by

$$
V_{p}(r)=\left\{\begin{array}{l}
V_{S R}(r) \text { for } r<r_{c} \\
V_{L R}(r) \text { for } r \geq r_{c}
\end{array}\right.
$$

Here, $r_{c}$ is the point where two forms cross each other for the first time. The crossing points, i.e., $r_{c}$ for zinc and lead atoms, occur at 17.5 and 14.0 a.u., respectively. The short-range form for the electron scattering based on the free-electron gas exchange potential is

$$
V_{S R}(r)=\left\{\begin{array}{l}
0.0622 \ln r_{s}-0.096+0.018 r_{s} \ln r_{s}-0.02 r_{s}, \quad r_{s} \leq 0.7 \\
-0.1231+0.03796 \ln r_{s}, \quad 0.7<r_{s} \leq 10 \\
-0.876 r_{s}^{-1}+2.65 r_{s}^{-3 / 2}-2.8 r_{s}^{-2}-0.8 r_{s}^{-5 / 2}, \quad 10 \leq r_{s}
\end{array}\right.
$$

where $r_{s}=[3 / 4 \pi \rho(r)]^{1 / 3}, \rho(r)$ is the electron charge density of a target atom which for a spherically symmetric atom is given by

$$
\rho(r)=\frac{1}{4 \pi} \sum_{n} \sum_{l} N_{n l}\left|\phi_{n l}(r)\right|^{2}
$$

where $N_{n l}$ is the occupancy number of the orbital $(n, l)$. All the terms of the total interaction potential can be generated using the target charge density $\rho(r)$. The longrange form of the polarization potential is given by $V_{L R}(r)=-\alpha_{d} / 2 r^{4}$, where $\alpha_{d}$ is the static electric dipole polarizability. For present calculations, the value of $\alpha_{d}$ is taken [21] as 47.91 a.u. for zinc and 45.89 a.u. for lead. The exchange potential is taken to be modified semiclassically as given by Gianturco and Scialla [22] and the absorption potential is included via the semiempirical model absorption potential of Staszewski, Schwenke, and Truhlar [23]. We avoid repeating their expression here. The impact energy considered in the present study ranges from 2.0 to $200 \mathrm{eV}$ for both the zinc and the lead atom. The first inelastic threshold [24] for zinc and lead is at 4.01 and $4.64 \mathrm{eV}$, while their ionization thresholds are at 9.39 and $7.42 \mathrm{eV}$, respectively.

\section{RESULTS AND DISCUSSION}

\section{A. Electron scattering from a zinc atom}

The calculated differential cross section in the energy range 2.0 to $200 \mathrm{eV}$ is shown in Fig. 1. In these figures the solid curve corresponds to the DCS value obtained by using the real potential (RP) as the total projectile-target interaction, and the dashed curve corresponds to those obtained with the complex potential (CP) as the interaction. At low energy, there is no difference between the results obtained using either the real or the complex potential, but at higher impact energies there is a small difference between the two calculated values, especially at those scattering angles where the DCS exhibit minima and maxima in the curve. This feature is expected since at low energies the scattering is mainly elastic and there is almost no effect of the absorption on the DCS values, whereas at higher impact energies there is reduction in the DCS values - in particular, the structure of dips and humps (both in magnitude and width) changes when the absorption effects are switched on. In the Table I we present our phase shift as a function of $K$ for the first four partial waves $l=0$ to 3 . These values of $K$ correspond to 


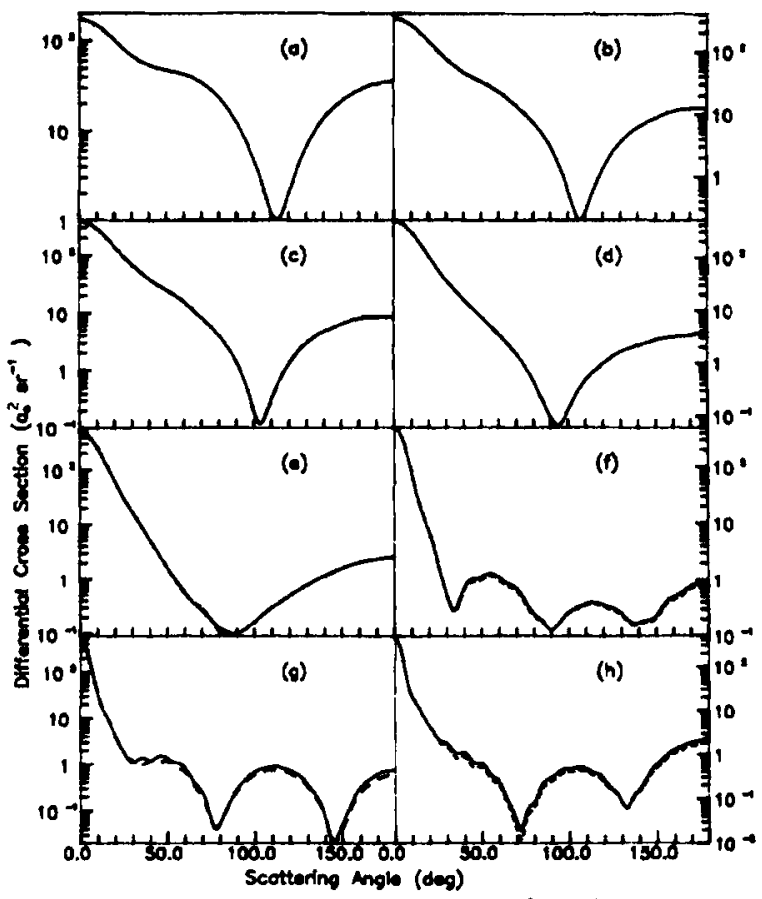

FIG. 1. Differential cross section in $\left(a_{0}^{2} \mathrm{sr}^{-1}\right)$ for electron scattering from zinc at (a) 2.0 , (b) 4.0 , (c) 5.0 , (d) 9.0 , (e) 14.0 , (f) $50.0,(\mathrm{~g}) 100$, and (h) $200 \mathrm{eV}$. Present calculations: —, with real potential; - - - with complex potential. energies for which experimental spin-polarization measurements for zinc are available. For higher partial waves (not shown here), the splitting between the spin-up and spin-down phase shifts is negligible.

Figure (2) shows the spin polarization $P(\vartheta)$ for zinc along with experimental points and the calculated values of McEachran and Stauffer (MS) [25]. Like DCS figures, here, also, all the solid curves correspond to values obtained using the RP and the dashed curves to values obtained using the CP in the Dirac equation. The present results show good agreement with the experimental data of Bartsch et al. [16] and also with the MS calculation in the low-energy region $(2-4 \mathrm{eV})$, i.e., before the onset of the inelastic threshold, while beyond this elastic region, our results show less satisfactory agreement with experiment. Further, we observe that the inclusion of the absorption potential in the interaction causes the minima to go deeper and the maxima to peak in general, but this feature is seen more clearly at higher impact energies (see Fig. 2). The two sets of calculations, i.e., MS and the present one, exhibit maxima and minima that are similar at about the same scattering angle, but different in magnitude. This difference indicates that the cross section and, consequently, the polarization parameters are sensitive to the choice of the interaction potential. As no measured values of $T$ and $U$ parameters are available, we have displayed, for the sake of completeness, the angular variation of parameters $U$ and $T$ in Fig. 3 at a few selected

TABLE I. Phase shifts (rad) with real potential of the first four partial waves for the elastic scattering of an electron from a zinc atom.

\begin{tabular}{ccccc}
\hline \hline$K$ & $\delta_{0}^{+}$ & $\delta_{1}^{+}$ & $\delta_{2}^{+}$ & $\delta_{3}^{+}$ \\
(a.u.) & $\delta_{0}^{-}$ & $\delta_{1}^{-}$ & $\delta_{3}^{-}$ \\
\hline 0.3834 & -1.2725 & -1.3816 & 0.5851 & 0.1942 \\
& -1.0482 & -1.2835 & 0.5883 & 0.1942 \\
0.4695 & -0.2635 & -2.1383 & 0.7013 & 0.2820 \\
& -2.0156 & -2.1383 & 0.7063 & 0.2820 \\
0.5422 & -1.1213 & & & \\
& -1.0617 & 0.1656 & 0.7605 & 0.3395 \\
0.6062 & 1.5954 & -2.9503 & 0.7676 & 0.3395 \\
& -0.6694 & -2.4457 & 0.7930 & 0.3804 \\
0.8133 & 1.6153 & -1.5544 & 0.8013 & 0.3804 \\
& 2.0468 & 0.6070 & 0.8040 & 0.4840 \\
1.0144 & 0.7077 & 2.6265 & 0.8150 & 0.4840 \\
& 0.8668 & 2.3568 & 0.7368 & 0.5668 \\
1.4850 & -0.1081 & 2.5855 & 0.7483 & 0.5668 \\
& -0.0109 & 0.7849 & 0.5658 & 0.7294 \\
1.9171 & -0.7034 & 0.8391 & 0.5764 & 0.7294 \\
& -0.5682 & 0.2956 & 0.4276 & 0.9260 \\
2.7113 & -0.9923 & 0.3323 & 0.4370 & 0.9262 \\
3.3207 & -2.8794 & -0.2827 & 0.2288 & 0.1477 \\
3.8345 & 1.2679 & -0.2460 & 0.2370 & 0.1478 \\
\hline \hline & 0.1992 & -0.7209 & 0.1071 & 0.2213 \\
& 1.7543 & -0.6695 & 0.1148 & 0.2217 \\
& 2.2368 & -1.2057 & 0.0176 & 0.0252 \\
\hline
\end{tabular}




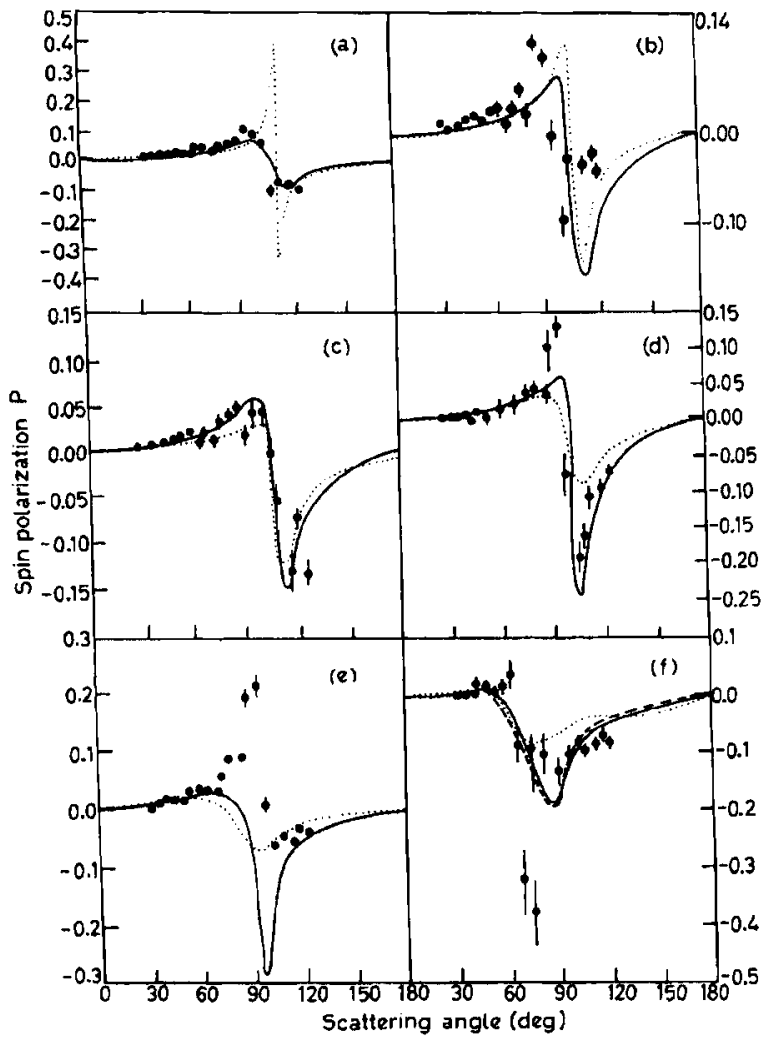

FIG. 2. Spin-polarization parameter $P$ for electrons scattered from zinc at (a) 2.0 , (b) 3.0 , (c) 4.0 , (d) 5.0 , (e) 9.0 , and (f) $14.0 \mathrm{eV}$. Present calculations: $\longrightarrow$, with real potential; $-\ldots$, with complex potential; ...., calculation of McEachran and Stauffer (Ref. [25]); 0 , experimental results (Ref. [16]).

energies. The parameter $U$ shows features similar to the spin-polarization $P$ curve.

\section{B. Electron scattering from a lead atom}

We have calculated the DCS, the spin polarization $P$, and the spin-polarization parameters $T$ and $U$ for electron scattering from lead similarly to the manner in which we have calculated them for the zinc atom having a closed-shell configuration. The present model is certainly a poorer approximation for an open-shell configuration like lead because it does not include the fine-structure effect. Figure 4 shows our DCS at collision energies between 2.0 and $200 \mathrm{eV}$. Here again, we display our results for both real and complex potentials. It is seen that the present model predicts the forward peaking, a number of minima and maxima at middle angles, and an enhanced backward slope. The influence of the absorption potential is seen only at higher impact energies. Its inclusion reduces the DCS both in magnitude and in width near the structure. Similar features were also observed for the case of a zinc atom. Phase shifts of the four lowest partial waves $(l=0$ to 3 ) for various impact

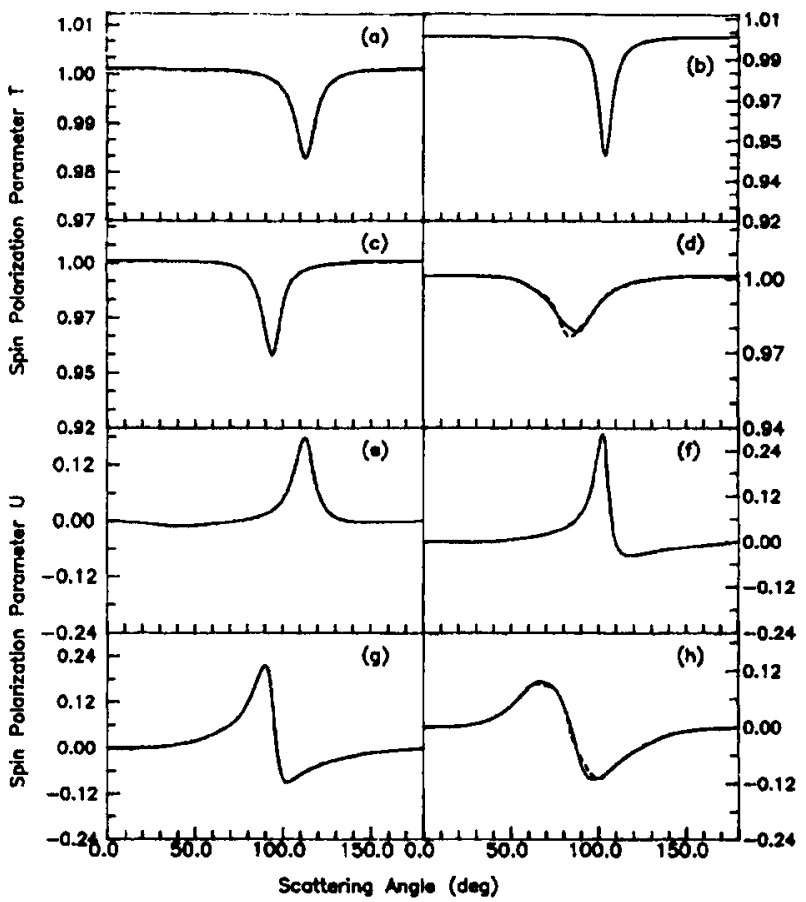

FIG. 3. The spin-polarization parameters $T$ and $U$ for electrons scattered from zinc with real and complex potentials at (a) 2.0, (b) 5.0 , (c) 9.0 , (d) 14.0 , (e) 2.0 , (f) $5.0,(\mathrm{~g}) 9.0$, and (h) $14.0 \mathrm{eV}$.

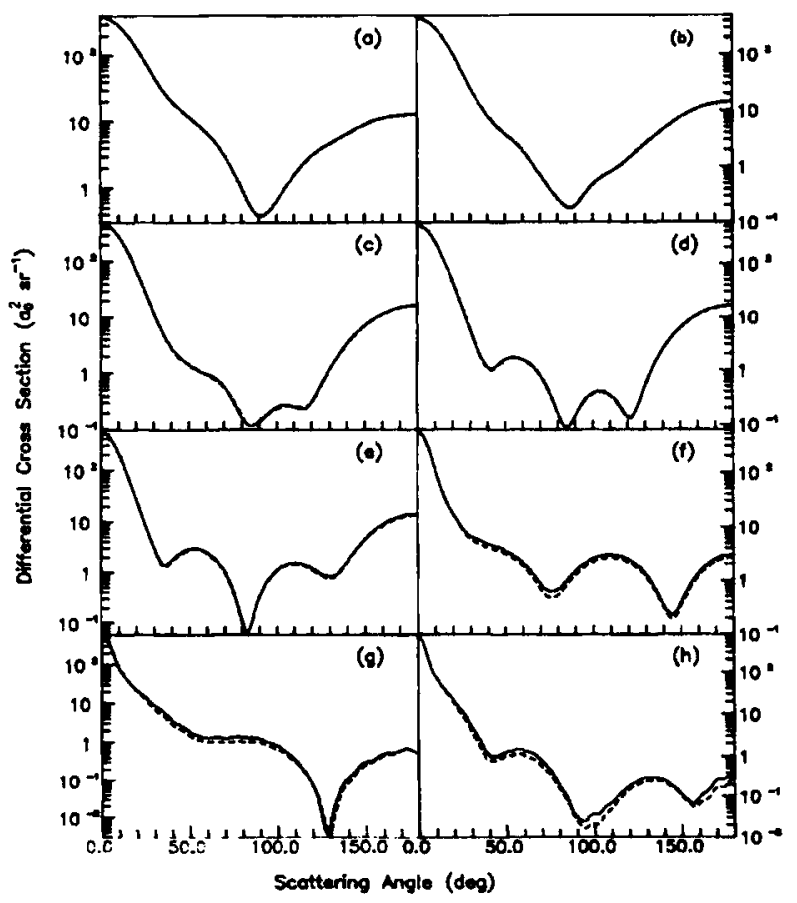

FIG. 4. Differential cross sections in $\left(a_{0}^{2} s r^{-1}\right)$ for electron scattering from lead at (a) 6.0, (b) 9.0, (c) 12.5 , (d) 17.0 , (e) 24.0, (f) 50.0, (g) 100.0, and (h) $180.0 \mathrm{eV}$. Present calculations: with real potential; --- , with complex potential. 
TABLE II. Phase shifts (rad) with real potential of the first four partial waves for the elastic scattering of an electron from a lead atom.

\begin{tabular}{|c|c|c|c|c|}
\hline $\begin{array}{c}K \\
\text { (a.u.) } \\
\end{array}$ & $\begin{array}{l}\delta_{0}^{+} \\
\delta_{0}^{-} \\
\end{array}$ & $\begin{array}{l}\delta_{1}^{+} \\
\delta_{l}^{-} \\
\end{array}$ & $\begin{array}{l}\delta_{2}^{+} \\
\delta_{2}^{-} \\
\end{array}$ & $\begin{array}{l}\delta_{3}^{+} \\
\delta_{3}^{-} \\
\end{array}$ \\
\hline 0.3834 & $\begin{array}{l}-1.4374 \\
-2.5865\end{array}$ & $\begin{array}{r}-2.3863 \\
0.9494\end{array}$ & $\begin{array}{l}0.5105 \\
0.5699\end{array}$ & $\begin{array}{l}0.1827 \\
0.1827\end{array}$ \\
\hline 0.6641 & $\begin{array}{l}1.3105 \\
1.8272\end{array}$ & $\begin{array}{r}1.0206 \\
-0.3032\end{array}$ & $\begin{array}{l}0.5303 \\
0.6369\end{array}$ & $\begin{array}{l}0.5164 \\
0.5164\end{array}$ \\
\hline 0.8133 & $\begin{array}{l}0.6326 \\
0.8637\end{array}$ & $\begin{array}{r}3.1783 \\
-2.5203\end{array}$ & $\begin{array}{l}0.4511 \\
0.5622\end{array}$ & $\begin{array}{l}0.6616 \\
0.6619\end{array}$ \\
\hline 0.8991 & $\begin{array}{l}0.3990 \\
0.5811\end{array}$ & $\begin{array}{l}2.1747 \\
1.4632\end{array}$ & $\begin{array}{l}0.3978 \\
0.5067\end{array}$ & $\begin{array}{l}0.7573 \\
0.7580\end{array}$ \\
\hline 0.9585 & $\begin{array}{l}0.2644 \\
0.4279\end{array}$ & $\begin{array}{l}2.7616 \\
2.3533\end{array}$ & $\begin{array}{l}0.3597 \\
0.4666\end{array}$ & $\begin{array}{l}0.8307 \\
0.8320\end{array}$ \\
\hline 1.0144 & $\begin{array}{l}0.1499 \\
0.3028\end{array}$ & $\begin{array}{l}1.4756 \\
3.7333\end{array}$ & $\begin{array}{l}0.3243 \\
0.4299\end{array}$ & $\begin{array}{l}0.9027 \\
0.9091\end{array}$ \\
\hline 1.1117 & $\begin{array}{l}0.0436 \\
0.1013\end{array}$ & $\begin{array}{l}1.0986 \\
2.3114\end{array}$ & $\begin{array}{l}2.6171 \\
0.3637\end{array}$ & $\begin{array}{l}1.0768 \\
1.0825\end{array}$ \\
\hline 1.3282 & $\begin{array}{l}-0.4204 \\
-0.2589\end{array}$ & $\begin{array}{l}0.6185 \\
1.2195\end{array}$ & $\begin{array}{l}1.3827 \\
0.2348\end{array}$ & $\begin{array}{l}1.6464 \\
1.6639\end{array}$ \\
\hline 1.9171 & $\begin{array}{l}-2.6626 \\
-1.8129\end{array}$ & $\begin{array}{r}-0.1167 \\
0.2205\end{array}$ & $\begin{array}{l}-0.2118 \\
-0.1205\end{array}$ & $\begin{array}{l}0.0462 \\
1.8147\end{array}$ \\
\hline 2.7113 & $\begin{array}{l}1.8658 \\
2.7707\end{array}$ & $\begin{array}{l}-1.1356 \\
-0.5615\end{array}$ & $\begin{array}{l}-0.7685 \\
-0.6417\end{array}$ & $\begin{array}{l}-0.5094 \\
-0.1494\end{array}$ \\
\hline 3.3207 & $\begin{array}{l}0.6053 \\
0.8257\end{array}$ & $\begin{array}{r}0.7256 \\
-1.4892\end{array}$ & $\begin{array}{l}-1.4555 \\
-1.2234\end{array}$ & $\begin{array}{l}-2.9852 \\
-2.7200\end{array}$ \\
\hline 3.6377 & $\begin{array}{l}0.3046 \\
0.4727\end{array}$ & $\begin{array}{l}-1.8166 \\
-2.5648\end{array}$ & $\begin{array}{l}-2.0736 \\
-1.6982\end{array}$ & $\begin{array}{l}-3.0053 \\
-2.7372\end{array}$ \\
\hline 3.8345 & $\begin{array}{l}0.2057 \\
0.3629\end{array}$ & $\begin{array}{r}2.7428 \\
-3.2610 \\
\end{array}$ & $\begin{array}{l}-2.2384 \\
-1.8215\end{array}$ & $\begin{array}{l}-2.4180 \\
-2.2357 \\
\end{array}$ \\
\hline
\end{tabular}

energies of electrons are presented in Table II.

Figure 5 shows our angular distribution of the spin polarization $P$ in the energy range $6.0-180 \mathrm{eV}$. The solid and dashed curves show our results with real and complex potentials, respectively. We also displayed on the curve the experimental results of Kaussen et al. [26] along with the theoretical calculation of Haberland and Fritsche [13] using a GKS approach and the $R$-matrix calculations of Bartschat [27]. The Bartschat calculation includes Mott scattering and the fine-structure effect. The results of this calculation are available at only two energies, i.e., 6.0 and $9.0 \mathrm{eV}$. The agreement between the

TABLE III. Calculated values of the integrated elastic $\left(\sigma_{\mathrm{el}}\right)$, total $\left(\sigma_{1}\right)$, and momentum-transfer $\left(\sigma_{m}\right)$ cross sections (in units of $\left.a_{0}^{2}\right)$ for the elastic scattering of an electron from a zinc atom. RP corresponds to the use of a real potential, $\mathrm{CP}$ to the use of a complex potential.

\begin{tabular}{|c|c|c|c|c|c|}
\hline \multirow{2}{*}{$\begin{array}{c}E \\
(\mathrm{eV}) \\
\end{array}$} & \multicolumn{2}{|c|}{$\sigma_{\mathrm{et}}$} & \multirow[b]{2}{*}{$\sigma_{1}$} & \multicolumn{2}{|c|}{$\sigma_{m}$} \\
\hline & RP & $\mathrm{CP}$ & & RP & $\mathrm{CP}$ \\
\hline 2.0 & 354.07 & 354.07 & 354.07 & 203.35 & 203.35 \\
\hline 3.0 & 322.39 & 322.89 & 322.89 & 137.00 & 137.00 \\
\hline 4.0 & 284.09 & 284.09 & 284.09 & 96.25 & 96.25 \\
\hline 5.0 & 149.24 & 149.24 & 149.24 & 70.66 & 74.66 \\
\hline 9.0 & 157.92 & 157.92 & 157.92 & 28.15 & 18.15 \\
\hline 14.0 & 103.97 & 103.80 & 104.17 & 13.74 & 13.72 \\
\hline 30.0 & 50.34 & 49.32 & 52.34 & 5.40 & 5.23 \\
\hline 50.0 & 34.89 & 33.50 & 37.58 & 5.20 & 4.25 \\
\hline 100.0 & 25.26 & 23.72 & 27.74 & 6.14 & 5.24 \\
\hline 150.0 & 21.38 & 19.91 & 23.38 & 5.70 & 5.08 \\
\hline 200.0 & 18.65 & 17.40 & 20.48 & 5.55 & 4.62 \\
\hline
\end{tabular}




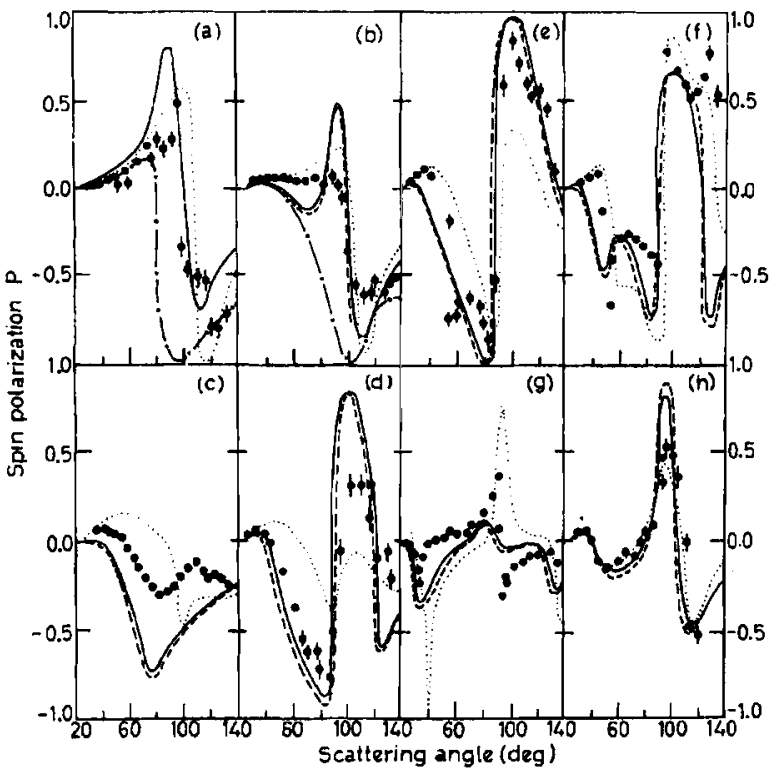

FIG. 5. Spin polarization $P$ for electrons scattered from lead at (a) 6.0 , (b) 9.0 , (c) 11.0, (d) 12.5, (e) 14.0, (f) 17.0, (g) 24.0, and (h) $180.0 \mathrm{eV}$. Present calculations: _-, with real potential; --- , with complex potential; . . . , calculation of Haberland and Fritsche (Ref. [13]); -.-..-., calculation of Bartschat (Ref. [27]); O O, experimental results (Ref. [26]).

present calculation and experimental results is by and large quite satisfactory at most of the energies. The results of Haberland and Fritsche [13] are similar to our results, but the magnitudes of the maxima and minima are different. In general, the structure of the polarization curve at all energies is better reproduced by the calculation of Haberland and Fritsche. At higher impact energies, present calculations agree well with the results of

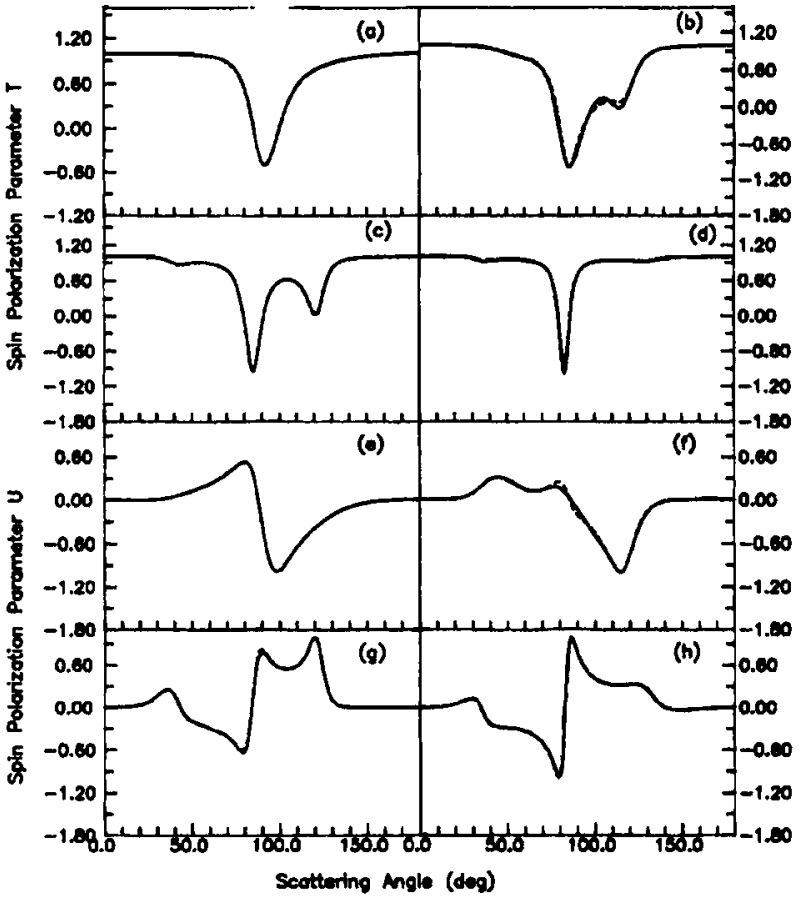

FIG. 6. The spin-polarization parameters $T$ and $U$ for electrons scattered from lead with real and complex potentials at (a) 6.0 , (b) 12.5 , (c) 17.0 , (d) 24.0 , (e) 6.0 , (f) 12.5 , (g) 17.0 , and (b) $24.0 \mathrm{eV}$.

Haberland and Fritsche. On the other hand, the calculations of Bartschat at 6 and $9 \mathrm{eV}$ show considerable variations in magnitude compared with our results and those of Haberland and Fritsche and measured values. No experimental values of $U$ and $T$ parameters are available presently, and, therefore, their angular variations are presented only at a few selected energies in Fig. 6.

TABLE IV, Calculated values of the integrated elastic $\left(\sigma_{e t}\right)$, total $\left(\sigma_{t}\right)$, and momentum-transfer $\left(\sigma_{m}\right)$ cross sections (in units of $\left.a_{0}^{2}\right)$ for the elastic scattering of an electron from a lead atom. RP corresponds to the use of a real potential, CP to the use of a complex potential.

\begin{tabular}{|c|c|c|c|c|c|}
\hline \multirow{2}{*}{$\begin{array}{c}E \\
(\mathrm{eV})\end{array}$} & \multicolumn{2}{|c|}{$\sigma_{\text {el }}$} & \multirow{2}{*}{$\begin{array}{c}\sigma_{t} \\
\left(a_{0}^{2}\right)\end{array}$} & \multicolumn{2}{|c|}{$\sigma_{m}$} \\
\hline & $\mathbf{R P}$ & CP & & RP & $\mathrm{CP}$ \\
\hline 2.0 & 384.58 & 384.58 & 384.58 & 206.30 & 206.30 \\
\hline 6.0 & 202.44 & 202.44 & 202.44 & 65.23 & 65.23 \\
\hline 9.0 & 149.58 & 149.57 & 149.62 & 42.72 & 42.72 \\
\hline 11.0 & 129.30 & 129.13 & 129.57 & 36.35 & 36.28 \\
\hline 12.5 & 118.35 & 117.97 & 118.91 & 33.58 & 33.39 \\
\hline 14.0 & 109.95 & 109.32 & 110.68 & 29.23 & 29.00 \\
\hline 17.0 & 98.20 & 97.03 & 99.82 & 31.73 & 31.24 \\
\hline 24.0 & 83.99 & 81.73 & 86.88 & 29.72 & 28.05 \\
\hline 50.0 & 54.22 & 60.57 & 58.51 & 23.32 & 19.38 \\
\hline 100.0 & 52.19 & 47.78 & 55.10 & 9.23 & 7.52 \\
\hline 150.0 & 45.42 & 41.35 & 47.70 & 5.36 & 4.26 \\
\hline $\begin{array}{l}180.0 \\
200.0\end{array}$ & $\begin{array}{c}41.81 \\
39.64\end{array}$ & $\begin{array}{c}38.03 \\
35.95\end{array}$ & $\begin{array}{l}43.87 \\
41.52\end{array}$ & $\begin{array}{r}4.50 \\
4.40\end{array}$ & $\begin{array}{r}3.54 \\
3.30\end{array}$ \\
\hline
\end{tabular}




\section{Elastic- and total-scattering cross sections}

The values of the present integrated elastic, total, and momentum-transfer cross sections for zinc and lead atoms are compiled in Tables III and IV. The elastic cross sections are obtained using both real and complex potentials. It is seen that the elastic cross sections with real potentials are larger than those obtained with complex potentials at all energies. This is not unexpected because the inclusion of absorption interaction reduces the DCS and, consequently, the elastic cross sections. The total cross sections and momentum-transfer cross sections are also presented in Tables III and IV. It is observed that total cross section descends rapidly from a few eV to $50.0 \mathrm{eV}$ and thereafter slowly with increasing impact energies. The momentum-transfer cross sections obtained using both real and complex potentials show a minima at $50.0 \mathrm{eV}$ for zinc and at $14.0 \mathrm{eV}$ for lead. The calculated values with real potentials are higher than those obtained with complex potentials. Again, the reduction in the momentum-transfer cross sections is due to the inclusion of absorption effect.

\section{CONCLUSIONS}

We have presented the results of our relativistic, totalscattering, momentum-transfer cross sections, DCS and the angular distribution of spin polarization $P$, and spinpolarization parameters $T$ and $U$ for electrons scattered from zinc and lead atoms. We compared our theoretical calculations with the experimental spin-polarization data. We showed that the present relativistic model is capable of explaining the features of the electron spin polarization when compared with the measured values of the asym- metry parameter. The agreement seems to be better at higher impact energies. For zinc, the complex part (the absorption potential) of the optical potential has no effect on the polarization parameters except that only its addition above the inelastic threshold has a discernable effect (see Fig. 2), but the same is more significant for lead. Thus, a better representation of the absorption potential which accounts for the combined effect of all the inelastic channels is desirable. An $a b$ initio calculation of the absorption potential is still an open problem. Here we employ a semiempirical absorption potential (see Ref. [23]) for electrons scattering which is obtained by treating the target gas as a homogeneous free-electron gas system. A detailed discussion on this aspect can be found in a paper by Thirumalai, Staszewska, and Truhlar [28]. The electron scattering from the heavier-species lead shows a significant amount of spin polarization compared to zinc. The present calculated DCS curves have features very similar to the theoretical and experimental results of the electron-cadmium process [25]. The difference between the present model calculations and those of others can be said to be due basically to the different choices of both the correction potential and the charge density which correctly simulate the required dynamical effects of the scattering process.

\section{ACKNOWLEDGMENTS}

This work has been supported by the Council of Scientific and Industrial Research (CSIR), New Delhi, India. Also, one of us (A.K.J.) wishes to acknowledge financial support from the Department of Science and Technology (DST), New Delhi, India.
[1] J. Kessler, Adv. At. Mol. Opt. Phys. 27, 81 (1991); see also Polarized Electrons, 2nd ed. (Springer-Verlag, Berlin, 1985).

[2] G. F. Hanne, Phys. Rep. 95, 95 (1983); see also Coherence in Atomic Collision Physics, edited by H. J. Beyer, K. Blum, and R. Hippler (Plenum, New York, 1988), p. 41.

[3] N. F. Mott, Proc. R. Soc. London, Ser. A 124, 425 (1929); 135, 429 (1932); see also N. F. Mott and H. S. W. Massey, The Theory of Atomic Collision (Oxford University, Oxford, 1987), p. 227

[4] D. W. Walker, Adv, Phys. 20, 257 (1971); J. Phys. B 3, 788 (1970).

[5] L. T. Sin Fai Lam, J. Phys. B 15, 119 (1982).

[6] B. Awe, F. Kemper, F. Rosicky, and R. Feder, Phys. Rev. Lett. 46, 603 (1983); J. Phys. B 16, 603 (1983).

[7] L. Fritsche, J. Noffke, and H. Gollisch, J. Phys. B 17, 1637 (1984).

[8] F. Kemper, F. Rosicky, and R. Feder, J. Phys. B 18, 1233 (1985).

[9] R. P. McEachran and A. D. Stauffer, J. Phys. B 19, 3523 (1986).

[10] K. Hasenburg, D. H. Madison, K. Bartschat, and $\mathbf{K}$. Blum, J. Phys. B 19, 1803 (1986).
[11] Sultana N. Nahar and J. M. Wadehra, Phys. Rev. A 43, 1275 (1991); see also ibid. 35, 2051 (1987).

[12] Sultana N. Nahar, Phys. Rev. A 43, 2223 (1991).

[13] R. Haberland and L. Fritsche, J. Phys. B 20, 121 (1987).

[14] K. Bartschat, J. Phys. B 20, L815 (1987).

[15] K. Bartschat, H.-J. Goerss, and R.-P. Nordbeck, Z. Phys. D 17, 25 (1990).

[16] M. Bartsch, H. Geesmann, G. F. Hanne, and J. Kessler, J. Phys. B 25, 1511 (1992).

[17] C. J. Joachain, Quantum Collision Theory (North-Holland, Amsterdam, 1983), Chap. 18.

[18] E. Clementi and C. Roetti, At. Data Nucl. Data Tables 14, 177 (1974).

[19] A. D. McLean and R. S. McLean, At. Data Nucl. Data Tables 26, 232 (1981).

[20] J. K. O'Connell and N. F. Lane, Phys. Rev. A 27, 1893 (1983).

[21] T. M. Miller, CRC Handbook of Chemistry and Physics (Chemical Rubber, Boca Raton, FL, 1984).

[22] F. A. Gianturco and S. Scialla, J. Phys. B 20, 3171 (1987).

[23] G. Staszewska, D. W. Schwenke, and D. G. Truhlar, Phys. Rev. A 29, 3078 (1984)

[24] C. E. Moore, Atomic Energy Levels, Natl. Bur. Stand. 
(U.S.) Circ. No. 467 (U.S. GPO, Washington, DC, 1949).

[25] R. P. McEachran and A. D. Stauffer, J. Phys. B 25, 1527 (1992).

[26] F. Kaussen, H. Geesmann, G. F. Hanne, and J. Kessler, J.
Phys. B 20, 151 (1987).

[27] K. Bartschat, J. Phys. B 18, 2519 (1985).

[28] D. Thirumalai, G. Staszewska, and D. G. Truhlar, Comments At. Mol. Phys. 20, 217 (1987). 